\title{
Action larvaire de Sitona lineatus L. sur quelques facteurs de production du pois protéagineux (Pisum Sativum L.)
}

\author{
P. Cantot \\ INRA Laboratoire de Zoologie, 86600 Lusignan, France
}

(reçu le 19 mai 1987, accepté le 11 mai 1989)

Résumé - Des expériences réalisées en conditions contrôlées (culture hydroponique en salle climatisée) ou semicontrôlées (culture de plein champ avec infestation artificielle sous cages) démontrent l'action des larves de Sitona lineatus $L$. sur quelques facteurs de production de pois protéagineux, souvent dès le seuil de 10 œufs par plante. Cette action se manifeste surtout sur le nombre et le poids de grains par plante. Dans le cas de la variété "Finale", c'est surtout la productivité des ramifications qui est affectée. La production de matière azotée totale par plante est significativement plus faible dans les lots infestés.

Sitona lineatus - Pisum sativum - facteur de production - larve

Summary - Effects of the larvae of Sitona lineatus L. on some productivity factors in proteaginous pea (Pisum sativum L.). Experiments in controlled conditions (hydroponic cultivation in an air-conditioned room) or semifield conditions (artificial infestation under cages in a field with 1 or 3 plants per cage) show the effect of the larvae of Sitona lineatus $L$. on some productivity factors in the proteaginous pea, at a threshold of 10 eggs per plant on isolated plants or 20 eggs per plant for groups of 3 plants. This effect is observed via the number of seeds and yield per plant. In the case of the cultivar "Finale" the effect is mainly observed on branches. With a nutritive solution without nitrogen the quality of the grain (nitrogen percentage) is diminished when the larvae destroy the nodules. The production of total nitrogen per plant is significantly less in infested plants in field conditions. The relationship between the number of larvae per plant and the percentage of nodules destroyed was confirmed $(r=0.96)$.

Sitona lineatus - Pisum sativum - production factor - larva

\section{INTRODUCTION}

Parmi les ravageurs du pois protéagineux, le sitone du pois (Sitona lineatus L.) est un des insectes les plus fréquents aussi bien en France (Bournoville, 1984; Cantot, 1986, 1987) que dans d'autres pays européens producteurs de pois : Grande-Bretagne (Bardner et al., 1979), Allemagne (Raiser, 1983). Les adultes dévorent le bord des folioles et stipules de façon caractéristique. Cette alimentation imaginale n'a que peu d'influence sur le rendement du pois (George, 1982), mais cependant ce type de déprédation peut servir à l'établissement d'une notation symptômatique permettant d'apprécier les populations d'adultes présentes au champ (Cantot, 1986).
La nuisibilité de $S$. lineatus est tout autant le fait de l'alimentation larvaire aux dépens des nodosités fixatrices d'azote qui se trouvent sur le système racinaire. La mise en évidence de l'action de ces larves sur les facteurs de production de la plante est délicate à réaliser par la mise en œuvre d'essais insecticides de plein champ, car d'une part il s'agit d'une action indirecte et d'autre part l'application d'insecticides peut supprimer d'autres éléments de la biocénose. Néanmoins, un certain nombre d'expérimentations utilisant les insecticides ont été réalisées par différents auteurs et tendent à prouver, notamment en Grande-Bretagne (Bardner et al., 1979; King, 1981) et en Belgique (Vulsteke et Seutin, 1985), la nuisibilité larvaire du sitone du pois. II est tou- 
tefois difficile dans des conditions de parcelles expérimentales de relier avec certitude un rendement et une population de ravageur, car les résultats sont variables d'une année à l'autre (King, 1981). Pour notre part, dans le cadre des recherches qui font l'objet de cette mise au point, nous avons réalisé des essais en conditions contrôlées ou semi-contrôlées en infestant des plantes avec des nombres connus d'œufs de $S$. lineatus. Le pourcentage d'éclosion de ces œufs étant voisin de $100 \%$, nous n'avons pas utilisé pour nos infestations de larves néonates, beaucoup plus difficiles à manipuler.

\section{MATÉRIEL ET MÉTHODES}

Afin de bien cerner les facteurs de production modifiés par l'alimentation larvaire des sitones, une première expérimentation a été menée en salle climatisée, puis les expériences ultérieures ont été réalisées en plein champ en tenant compte de la compétition entre plantes.

\section{En salle climatisée}

Les pois (variété “Finale») sont cultivés dans des pots plastique $(11 \mathrm{~cm} \times 11 \mathrm{~cm} \times 12 \mathrm{~cm})$, dont les trous de drainage sont grillagés finement mais permettent le passage des radicelles tout en empêchant celui des larves. Chaque pot est rempli de vermiculite expansée et les pois (1 plante par pot) implantés dans ce milieu sont inoculés avec une suspension de Rhizobium leguminosarum, qui va induire la formation de nodosités sur les racines. Les plantes sont ensuite arrosées toutes les semaines avec une solution nutritive (milieu Lefèvre) qui ne contient pas d'éléments azotés; sa composition est indiquée Tableau I. Celle-ci n'est pas directement versée dans le pot de culture mais mise dans un contenant où viennent plonger les radicelles, afin que la vermiculite ne soit pas saturée d'humidité.

L'expérience a été réalisée à une température de $20^{\circ} \mathrm{C}$ et à une photophase de $16 \mathrm{~h}$. Lorsque les pois atteignent le stade 3-4 feuilles, on place à leur base des cufs de $S$. lineatus provenant de la ponte d'adultes élevés sur des brins coupés de pois, selon une technique décrite par Newton (1958). Le nombre d'œufs par plante a varié, selon les modalités expérimentales, d'un minimum de 5 à un maximum de 50 , les témoins n'étant pas infestés. Nous avons effectué

Tableau I. Composition de la solution nutritive pour 1 litre d'eau.

Macro-éléments

\begin{tabular}{llll}
\hline $\mathrm{KH}_{2} \mathrm{PO}_{4}$ & $0,140 \mathrm{~g}$ & $\mathrm{H}_{3} \mathrm{BO}_{3}$ & $2,00 \mathrm{mg}$ \\
$\mathrm{Mg} \mathrm{SO}_{4}, 7 \mathrm{H}_{2} \mathrm{O}$ & $0,190 \mathrm{~g}$ & $\mathrm{Mn} \mathrm{SO}_{4}, \mathrm{H}_{2} \mathrm{O}$ & $1,80 \mathrm{mg}$ \\
$\mathrm{Ca} \mathrm{Cl}_{2}$ & $0,220 \mathrm{~g}$ & $\mathrm{Zn} \mathrm{SO}$ & \\
$\mathrm{K}_{2} \mathrm{SO}_{4}$ & $0,070 \mathrm{~g}$ & $\mathrm{Na}_{2} \mathrm{Mo} \mathrm{O}_{4}, 2 \mathrm{H}_{2} \mathrm{O}$ & $0,20 \mathrm{mg}$ \\
$\mathrm{Fe}_{2} \mathrm{O}_{3}$ & $0,015 \mathrm{~g}$ & $\mathrm{Co}\left(\mathrm{NO}_{3}\right)_{2}, 6 \mathrm{H}_{2} \mathrm{O}$ & traces \\
\hline
\end{tabular}

25 répétitions par modalité. Un peu plus de 2 mois sont nécessaires pour arriver à la maturation des pois, qui sont récoltés puis mis à sécher dans un abri ventilé. Les plantes sont ensuite examinées une par une et différents facteurs de production sont mesurés : hauteur de la plante, nombre total de grains par plante, poids de 1000 grains par plante, nombre de ramifications par plante, nombre total de grains sur les ramifications. Pour chaque plante, l'ensemble des grains est broyé, puis la matière azotée totale (MAT) est mesurée selon la technique de Kjeldahl.

\section{En plein champ}

Nous avons utilisé la même variété qu'en salle climatisée. Au moment de la levée du pois (stade "crosse"), et surtout avant l'arrivée des adultes de sitones hivernants qui vont contaminer la culture, on isole, selon les expérimentations, 1 à 3 plantes dans un cylindre de PVC (diamètre $14 \mathrm{~cm}$, hauteur $10 \mathrm{~cm}$ ) qui est enfoncé dans le sol de 5 à $6 \mathrm{~cm}$. Sur le bord extérieur est fixé un bourrelet en mousse de polyuréthane, qui assure la jonction avec une bonnette en grillage polyester (mailles de 0,8 mm) mesurant $1 \mathrm{~m}$ de haut et maintenue par 2 tuteurs. Lorsque la plantule de pois possède 3 à 4 feuilles, on dispose les œufs selon les mêmes modalités que dans l'expérimentation menée en salle climatisée. L'inoculation avec des bactéries du genre Rhizobium n'est pas nécessaire, car elles se trouvent à l'état naturel dans les champs. La récolte intervient là aussi environ 2 mois après les infestations et les facteurs de production sont notés plante à plante, selon les mêmes techniques que celles décrites précédemment. En plein champ, beaucoup de facteurs interviennent sur la productivité des pois et notamment la compétition entre plantes. Nous avons donc, selon les années, pratiqué différentes modalités expérimentales.

Dans la première expérience, on dispose 1 plante par bonnette, celle-ci étant prise au hasard dans une parcelle de grande culture ensemencée en pois. Pour la seconde expérience, on dispose là aussi 1 plante par bonnette, mais le reste de la parcelle est laissé en sol nu. La troisième expérience se compose de 3 plantes par bonnette, ces plantes étant prises au hasard dans une parcelle de grande culture ensemencée en pois. Dans tous les cas, le nombre de répétitions est de 25 par modalité d'infestation.

\section{RÉSULTATS}

En salle climatisée, lorsque le pois ne dispose que de sa nodulation pour lui fournir l'azote nécessaire à sa croissance, tous les facteurs de production examinés sont significativement affectés par l'alimentation larvaire des sitones, sauf le poids de 1000 grains. La Figure 1 récapitule les résultats obtenus. Les nombres de grains et les poids de grains par plante sont significativement inférieurs pour les pois infestés, dès le seuil de 10 œufs par plante. Les pots avec 5 œufs par plante ne diffèrent pas du témoin, pour tous les paramètres examinés. La matière azotée totale produite par plante est significativement plus 


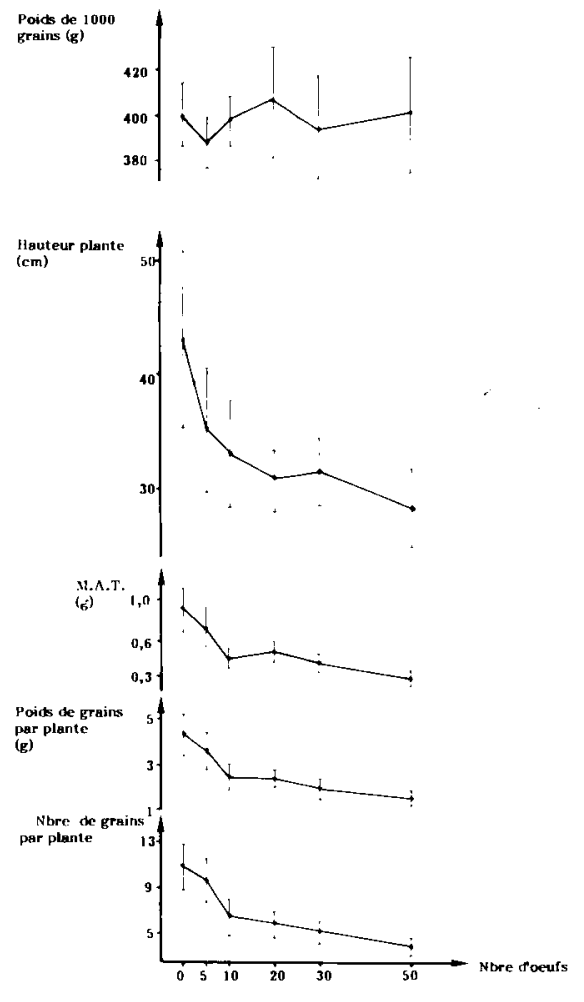

Fig. 1. Action larvaire de $S$. lineatus à différents niveaux d'infestation sur quelques critères de rendement du pois protéagineux (expérimentation en salle climatisée). Les barres verticales représentent l'intervalle de confiance de la moyenne. faible pour $10,20,30$ et 50 œufs par plante par rapport au témoin et à 5 œufs. Le niveau de production des plantes dans les conditions de la salle climatisée est réduit par rapport à l'extérieur : on soulignera en particulier que dans les conditions de salle climatisée il n'y a pas d'émission de ramifications secondaires.

Les principaux résultats acquis en plein champ sont récapitulés sur les Tableaux II, III et IV. La productivité en poids de grains par plante pour les témoins est variable selon les années. Pour l'expérience $n^{\circ} 2$, elle se situe à plus de $40 \mathrm{~g}$, alors qu'elle est beaucoup plus faible dans l'expérience $n^{\circ} 3$ avec environ $7 \mathrm{~g}$; il n'y a dans ce dernier cas que très peu de ramifications secondaires. Globalement, il existe souvent un effet dépressif dû aux sitones, mais il s'exprime différemment selon les années et les modalités expérimentales.

Ainsi, dans la première expérience, la hauteur, le nombre de grains, le poids de grains ainsi que la matière azotée totale produite par plante sont significativement plus faibles dans les lots infestés. L'interprétation statistique du poids de grains a été effectuée sur les données transformées en $\log$.

Dans la deuxième expérience, il n'existe pas, globalement, de différences significatives sur les

Tableau II. Action larvaire de $S$. lineatus à différents niveaux d'infestation sur quelques critères de rendement du pois protéagineux (expérimentation $n^{\circ} 1$ en plein champ).

\begin{tabular}{|c|c|c|c|c|c|c|c|}
\hline \multirow{2}{*}{$\begin{array}{l}\text { Facteurs de production } \\
\text { par plante }\end{array}$} & \multicolumn{5}{|c|}{ Modalité } & \multicolumn{2}{|c|}{ Analyse } \\
\hline & Témoin & $10 œ u f s$ & 25 œufs & 50 œufs & 100 œufs & $F$ & Signification \\
\hline Longueur totale en $\mathrm{cm}$ & $131 \mathrm{a}$ & $86^{b}$ & $100^{b}$ & $94 b$ & $102^{b}$ & 5,17 & HS \\
\hline Nbre de grains & $54 a$ & $39 b$ & $45^{b}$ & $42^{b}$ & $41 \mathrm{~b}$ & 4,10 & HS \\
\hline Poids de grains $(\mathrm{g})$ & $16,8^{a}$ & $12,1 \mathrm{~b}$ & $13,8 \mathrm{ab}$ & $13,0 \mathrm{~b}$ & $12,8^{b}$ & 3,58 & HS \\
\hline Poids de MAT produite (g) & $4,2^{a}$ & $3,1 \mathrm{~b}$ & $3,4^{b}$ & $3,1^{b}$ & $2,9 \mathrm{~b}$ & 5,11 & HS \\
\hline
\end{tabular}

Les chiffres suivis d'une même lettre sur une même ligne ne diffèrent pas significativement entre eux.

Tableau III. Action larvaire de $S$ lineatus à différents niveaux d'infestation sur quelques critères de rendement du pois protéagineux (expérimentation $n^{\circ} 2$ en plein champ).

\begin{tabular}{|c|c|c|c|c|c|c|c|c|}
\hline \multirow{2}{*}{$\begin{array}{l}\text { Facteurs de production } \\
\text { par plante }\end{array}$} & \multicolumn{6}{|c|}{ Modalité } & \multicolumn{2}{|c|}{ Analyse } \\
\hline & Témoin & 5 œuts & 10 œufs & 20 œufs & 30 œufs & 50 œufs & $F$ & Signification \\
\hline Longueur totale en $\mathrm{cm}$ & 189 & 181 & 155 & 188 & 178 & 165 & 1,98 & NS \\
\hline Nbre de grains & 106 & 106 & 94 & 107 & 105 & 96 & 1,86 & NS \\
\hline Poids de grains $(\mathrm{g})$ & 41,4 & 41,8 & 37,1 & 41,4 & 41,0 & 38,1 & 1,32 & NS \\
\hline Poids de MAT produite ( $\mathrm{g}$ ) & 10,7 & 10,9 & 9,8 & 10,8 & 10,9 & 10,0 & 0,88 & NS \\
\hline
\end{tabular}


Tableau IV. Action larvaire de $S$. lineatus à différents niveaux d'infestation sur quelques critères de rendement du pois protéagineux (expérimentation $\mathrm{n}^{\circ} 3$ en plein champ).

\begin{tabular}{|c|c|c|c|c|c|c|c|c|}
\hline \multirow{2}{*}{$\begin{array}{l}\text { Facteurs de production } \\
\text { par plante }\end{array}$} & \multicolumn{6}{|c|}{ Modalité } & \multicolumn{2}{|c|}{ Analyse } \\
\hline & Témoin & 5 œufs & 10 œufs & 20 œufs & 30 œufs & 50 œufs & $F$ & Signification \\
\hline Longueur totale en $\mathrm{cm}$ & $68 \mathrm{ab}$ & $74 a$ & $67 a b$ & 610 & $67 a b$ & $64 b$ & 2,64 & $\mathrm{~S}$ \\
\hline Nbre de grains & $22^{a}$ & $24 a$ & $22 a$ & $18 \mathrm{~b}$ & $18^{b}$ & $18^{b}$ & 5,62 & HS \\
\hline Poids de grains (g) & $7,2^{a}$ & $7,2^{a}$ & $6,9 a$ & $5,3^{b}$ & $5,6^{\mathrm{b}}$ & $5,8^{\mathrm{b}}$ & 6,07 & HS \\
\hline Poids de MAT produite (g) & $1,8 \mathrm{a}$ & $1,9 \mathrm{a}$ & $1,8^{a}$ & $1,3 \mathrm{~b}$ & $1,4 \mathrm{~b}$ & $1,4 \mathrm{~b}$ & 8,57 & HS \\
\hline
\end{tabular}

Les chiffres suivis d'une mème lettre sur une même ligne ne diffèrent pas significativement entre eux.

mêmes critères que ceux précédemment cités, bien que l'on observe dans tous les cas une baisse de productivité pour la modalité 10 œuis par plante.

Dans le cas de la troisième expérience, le nombre de grains, le poids de grains et la matière azotée et totale produite par plante sont affectés à partir de 20 œufs par plante. La productivité des plantes est très hétérogène au sein d'une même bonnette et les facteurs de rendement de cette expérimentation sont d'ailleurs calculés sur les moyennes de la production des 3 plantes, car l'on ne peut maîtriser l'homogénéité du déplacement des larves vers les nodosités.

\section{DISCUSSION}

En salle climatisée comme en plein champ, les facteurs de production du pois protéagineux sont donc affectés par les larves de $S$. lineatus, qui s'alimentent des nodosités fixatrices d'azote. La comparaison des différentes modalités montre que, selon le niveau de productivité atteint par les plantes, des compensations existent, mais elles se manifestent plus ou moins. Dans le cas où les plantes émettent des ramifications, ce sont surtout celles-ci qui vont être affectées. Si les plantes sont cultivées de façon isolée comme dans la $2^{\mathrm{e}}$ expérience, une baisse de production significative est seulement enregistrée sur les ramifications et ne va pas influer sur le rendement final de la plante. Par contre, si les plantes sont plus proches les unes des autres (expérience $n^{\circ} 1$ ), la productivité des ramifications sera prépondérante pour le rendement (Fig. 2). Enfin, dans la $3^{e}$ expérience en plein champ, où les plantes sont disposées par 3 dans chaque bonnette, il y a peu d'émission de ramifications secondaires. Cette situation n'est pas due aux sitones mais à la compétition élevée entre plantes et aux conditions climatiques de l'année, peu propices à un bon rendement. Néanmoins,

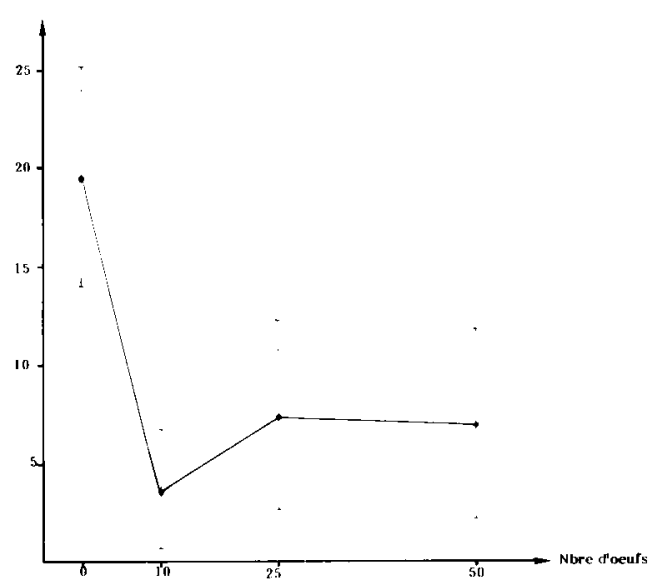

Fig. 2. Action larvaire de $S$. lineatus en plein champ sur la production des ramifications.

on note là aussi une baisse de productivité à partir de 10 œufs, ce qui correspond à une moyenne de 8 larves par plante avec une nodulation détruite à $80 \%$. II se dégage donc de ces 3 expérimentations la conclusion qu'un phénomène de compensation des pertes de productivité dues aux sitones existe en plein champ lorsque les plantes sont cultivées dans de très bonnes conditions, alors qu'en conditions de compétition entre plantes ou d'année moins favorable cette compensation n'existe plus.

On notera aussi que la compétition larvaire est importante à partir des niveaux d'infestation élevés, comme le prouvent les comptages effectués en plein champ, où les effectifs larvaires ne sont pas supérieurs malgré les accroissements de niveaux d'infestation d'œufs (Tableau V). Ce phénomène, déjà évoqué par Goldson et al. (1988) à propos de $S$. discoïdeus Gyll. sur la luzerne, est important à prendre en considération, mais probablement dépendant du nombre de nodosités disponibles, élément que l'on ne peut contrôler. On notera tout de même qu'il existe une relation hautement significative entre le nombre de larves présentes et le pourcentage de nodosités 
Tableau V. Développement des larves de $S$. lineatus et pourcentage de nodosités détruites en plein champ selon le niveau d'infestation.

\begin{tabular}{ccc}
$\begin{array}{c}\text { Nombre d'œufs } \\
\text { par plante }\end{array}$ & $\begin{array}{c}\text { Nombre de } \\
\text { larves par } \\
\text { plante }\end{array}$ & $\begin{array}{c}\text { Pourcentage de } \\
\text { nodosités } \\
\text { détruites }\end{array}$ \\
\hline & & \\
5 & 2,1 & 56,4 \\
10 & 5,7 & 58,2 \\
20 & 8,4 & 82,1 \\
30 & 6,9 & 88,8 \\
50 & 8,4 & 83,0 \\
\hline
\end{tabular}

détruites $(r=+0,96$ pour $39 \mathrm{ddl})$. Dans les conditions d'infestation naturelle, une relation identique a d'ailleurs déjà été démontrée (Cantot, 1987).

Dans toutes ces expérimentations en plein champ, 2 facteurs de production n'ont jamais été affectés : le poids de 1000 grains et le nombre de grains par gousse. Dans la majorité des cas, on trouve par contre une baisse significative de la matière azotée totale produite par plante. Ces résultats montrent que le mode d'action des sitones sur le pois est très différent de celui d'autres ravageurs, puisque, dans des conditions identiques de culture, le puceron du pois Acyrthosiphon pisum Harris agit sur le poids de 1000 grains, mais pas sur la teneur azotée de ceux-ci (Badenhausser, 1987).

\section{CONCLUSION}

Ces différentes études réalisées en conditions d'infestations artificielles prouvent l'action dommageable des larves de sitone sur quelques facteurs de production du pois protéagineux. En plein champ, c'est surtout la productivité des ramifications secondaires (lorsqu'elles sont présentes) qui exprime le mieux les différences dans le cas du cultivar «Finale». Mais d'autres variétés n'en possèdent pas (Raphalen, 1984) et l'expression des pertes demanderait à être étudiée. Le seuil de nuisibilité relativement peu élevé (10 ou 20 œufs par plante selon les expérimentations) se comprend par le fait qu'aucun ennemi naturel des œufs ou des larves n'intervient, alors qu'en conditions naturelles un certain nombre de parasites (Aeschlimann, 1977), de prédateurs (Rybchin, 1982) ou de champignons entomopathogènes (Riba, 1985) peuvent réguler les populations. Un des facteurs limitants doit être aussi le nombre de nodosités disponibles sur les racines, bien que les travaux récents (Aeschlimann, 1986) effectués sur $S$. discoideus Gyll. et
S. humeralis Steph., espèces qui se développent sur la luzerne, indiquent que l'alimentation larvaire aux dépens des nodosités n'est pas indispensable : pourtant, nous avons montré qu'il existe une corrélation hautement significative entre le nombre de nodosités dévorées et le nombre de larves de sitones par plante. Au-delà de 10 larves par plante, toutes les nodosités sont détruites au mois de juin (Cantot, 1986). Johnson \& O'Keefe (1981) ont d'ailleurs prouvé la digestion des Rhizobium par les larves de $S$. lineatus. Récemment, Wolfson (1987) a démontré dans le cas du trèfle (Trifolium pratense L.) et de la luzerne (Medicago sativa L.) la meilleure réussite du développement larvaire de Sitona hispidulus F. en présence de nodosités. Pour notre part, dans une expérimentation réalisée en salle climatisée, nous avons comparé la variété «Frisson» à son mutant isogénique non nodulant. Pour une infestation avec 20 œufs par plante et 25 répétitions, nous avons obtenu en moyenne 6 larves pour la variété contre 0 pour le mutant non nodulant.

II serait abusif d'extrapoler directement les résultats que nous avons obtenus aux conditions de la pratique agricole. En effet, le faible nombre d'œufs par plante pouvant causer les dommages peut inquiéter vu la prolificité des femelles de $S$. lineatus, qui peuvent pondre en moyenne 1000 œufs avec des maxima se situant aux alentours de 2000 . En fait, dans les conditions naturelles, cette ponte est très échelonnée dans le temps et tous les œuifs ne se développeront pas, car aux ennemis naturels cités plus haut vient s'ajouter l'influence des conditions climatiques et du stade de la plante hôte.

Dans les essais insecticides réalisés au champ par les auteurs cités dans l'introduction, les résultats significativement différents ne s'observent que lorsque le nombre de larves par plante est supérieur à 7 . Compte tenu de tous les facteurs de régulation, il est évident que cet effectif ne peut être atteint qu'avec une forte contamination des champs par les adultes. En France, d'après les enquêtes menées dans différentes régions productrices, cela ne concerne que 1 champ sur 6 (Bournoville, 1984). Ce "seuil» doit d'ailleurs être précisé et surtout tenir compte de l'état du végétal, comme le prouvent nos différentes expérimentations.

\section{REMERCIEMENTS}

Nous tenons à remercier $\mathrm{M}^{\mathrm{He}} \mathrm{N}$. Amarger (INRA, Dijon), qui nous a fourni les différentes souches de Rhizobium nécessaires pour les expérimentations en salle climatisée. Nos remerciements vont aussi à $R$. Bournoville (INRA, Lusignan) pour les corrections apportées au manuscrit ainsi qu'à Mie I. Badenhausser 
et J. Lerin (INRA, Lusignan) pour leur aide lors de l'interprétation statistique des résultats. M. R. Cousin (INRA, Versailles) a eu l'amabilité de nous fournir les graines de «Frisson" non nodulant.

\section{RÉFÉRENCES}

Aeschlimann J.P., (1977) Notes on Patasson lameerei (Hym. : Mymaridae) an egg parasitoid of Sitona spp. (Col. Curculionidae) in the mediterranean region. Entomophaga 22, 111-114

Aeschlimann J.P. (1986) Rearing and larval development of Sitona spp. (Coleoptera Curculionidae) on the root system of Medicago spp. plants (Leguminosae). Z. Angew. Entomol. 101, 461-469

Bardner R., Fletcher K.E. \& Griffiths D.C., (1979) Problems in the control of the pea and bean weevil (Sitona lineatus). Proc. Br. Crop. Prot. Conf, Pests and Dis. BCPC Bracknell, 223-229

Badenhausser I. (1987) Le puceron du pois (Acyrthosiphon pisum Harris) et le pois protéagineux de printemps. Conf. Int. Ravageurs Agric. ANPP, Paris 6, 323329

Bournoville R. (1984) Insectes nuisibles aux protéagineux, situation actuelle, organisation de lutte. Journ. Int. Protéagineux, Paris 12 déc. 1984, ITCF, Paris 199 217

Cantot P. (1986) Quantification des populations de Sitona lineatus $L$. et de leurs attaques sur pois protéagineux (Pisum sativum L.). Agronomie 6, 481-486

Cantot P. (1987) Influence de Sitona lineatus L. sur la nodulation et les facteurs de rendement du pois protéagineux. Colloq. Nutrition Azotée des Légumineuses, Versailles 19-21 Nov., 1985 INRA, Paris, 299304
George K.S. (1982) Root nodule damage by larvae of Sitona lineatus and its effects on yield of green peas. Plant Pathol. 2, 172-176

Goldson S.L., Frampton E.R. \& Proffitt J.R. (1988) Population dynamics and larval establishment of Sitona discoideus (Coleoptera : Curculionidae) in New Zealand lucerne. J. Appl. Ecol. 25, 177-195

Johnson M.P. \& O'Keeffe L.E. (1981) Presence and possible assimilation of Rhizobium leguminosarum in the gut of pea leaf weevil, Sitona lineatus, larvae. Entomol. Exp. Appl. 29, 103-108

King J.M. (1981) Experiments for the control of pea and weevil (Sitona lineatus) in peas, using granular and liquid insecticides. Crop Prot. Conf. Pests and Dis. BCPC Bracknell ed., 327-331

Newton R.C. (1958) Rearing Sitona hispidulus larvae for various research uses. J. Econ. Entomol. 51, $917-$ 918

Raiser E. (1983) Blattrandkäfer. Pflanzenschutz ABC 5,608

Raphalen J.L. (1984) Essais agro-météo pois de printemps, féverole de printemps. Résultats de 3 années d'expérimentation 1981, 1982, 1983. ITCF Paris

Riba G. (1985) Contribution à l'étude génétique de quelques hyphomycètes entomopathogènes. Thèse de Doctorat ès Sciences, Paris 6

Rybchin V.E. (1982) Enemies of root-nodule weevils. Zashch. Rast. 2. 30-31

Vulsteke G. \& Seutin E. (1985) Lutte contre le sitone du pois Sitona lineatus $L$. Meded. Fac. Landbouwwet., Rijksuniv. Gent. 50, 651-653

Wolfson J.L. (1987) Impact of Rhizobium nodules on Sitona hispidulus, the clover root curculio. Entomol. Exp. Appl. 43, 237-243 